\title{
Pituitary Adenoma Presenting as Anorexia Nervosa: A Case Report
}

\author{
Dhungana $\mathrm{S}^{1}$, Ojha $\mathrm{SP}^{2}$, Chapagai $\mathrm{M}^{3}$, Tulachan $\mathrm{P}^{4}$
}

1. Teaching Assistant, Dept. Of Psychiatry, Maharajgunj Medical Campus, Kathmandu 2. Assoc. Prof. Dept. Of Psychiatry, Maharajgunj Medical Campus, Kathmandu 3. Assoc. Prof. Dept. Of Psychiatry, Maharajgunj Medical Campus, Kathmandu 4. Lecturer, Dept. Of Psychiatry, Maharajgunj Medical Campus, Kathmandu

E-mail *Corresponding author: $\underline{\text { iomsaras@gmail.com }}$

\begin{abstract}
Anorexia nervosa is a syndrome characterized by severe weight loss and preoccupation of distorted body image with resulting complications due to starvation. In most of the typical cases of anorexia nervosa, neuroimaging is not done. However, with increasing evidence of intracranial lesions associated with eating disorders including anorexia nervosa, neuroimaging is indicated in almost all cases. We present a case of a 17-year-old female who was admitted initially with the diagnosis of anorexia nervosa (restricting type) and later found to have pituitary adenoma.
\end{abstract}

Keywords: Trichotillomania, Childhood Onset, Treatment

\section{INTRODUCTION}

Anorexia nervosa is a chronic disorder characterized by malnutrition and emaciation resulting from psychogenic aversion to food. ${ }^{1} \mathrm{It}$ is one of the most common psychiatric diseases among young women. ${ }^{2}$ Four diagnostic criteria for anorexia nervosa according to Diagnostic and Statistical Manual (DSM-IV) are refusal to maintain body weight at or above a minimally normal weight for age and height(weight loss less than $85 \%$ of expected); intense fear of gaining weight or becoming fat, even though underweight; disturbance in the way in which one's body weight or shape is experienced, undue influence of body weight or shape on self- evaluation, or denial of the seriousness of the current low body weight; and in postmenarchal women, amenorrhoea for at least three consecutive menstrual cycles. ${ }^{3}$ The illness usually occurs in girls within a few years of the menarche so that the most common age of onset is between 14 and 18 years. There are two main types of anorexia nervosa (restrictive and purging), and both have an impact on appetiteregulating hormones. Moreover, they lead to changes in the hypothalamic-pituitary-gonadal and hypothalamic-pituitary-adrenal axes, bone loss, and growth hormone resistance. ${ }^{4}$

A regular feature of the illness is its concealment and the avoidance of treatment. The patient's dread of fatness is so common that it is pathognomonic of anorexia nervosa, however, exceptions are there. Special mention is the occurrence of anorexia nervosa in eastern countries where thinness is not generally admired. The imposition of fear of fatness as a diagnostic criterion on patients from a different 
culture, where slimness is not valued, amounts to a failure to understand illness in the context of its culture. ${ }^{4}$ International Classification of Diseases (ICD-10) has therefore given the term atypical anorexia nervosa for such syndromes where patients do not fulfill all the criteria.

Occult intracranial tumors masquerading as early-onset anorexia nervosa have been reported. The possibility of organic disorder should always be considered before making a diagnosis of anorexia nervosa. This is particularly important if the presentation is atypical and the characteristic psychopathology is absent. ${ }^{5} \mathrm{We}$, hereby, report a case of pituitary adenoma who was initially diagnosed as a case of anorexia nervosa.

\section{CASE HISTORY}

A 17-year-old unmarried female high school student belonging to a family of low socioeconomic status from a remote village presented to our service with complaints of secondary amenorrhoea of two years duration, significant reduction in appetite and weight of over six months(more than $40 \%$ decrease in weight), nausea and vomiting, and low energy of one month duration. The patient was well adjusted till then and had her menarche at 13 years of age which was irregular in the first year and then became regular in the next year. She did not have history of galactorrhoea or sexual dysfunction. Other personal history was insignificant while family history revealed gastro- intestinal malignancy in second degree relative(maternal grandfather) and hematological malignancy in second degree relative (paternal uncle). Her great- grandfather on paternal side had suffered from dementia, and her paternal uncle had mood disorder (mania). Assessment of temperament revealed a quiet and easy going girl who adapted well in varying circumstances.

General physical examination revealed a conscious and alert girl with muscle wasting. Her body weight was 26 kilograms and height was 140 centimeters with a body mass index (BMI) of $13.2 \mathrm{~kg} / \mathrm{m}^{2}$. Secondary sexual characters were well developed. Her blood pressure was $80 / 60 \mathrm{mmHg}$ and pulse rate was 84 beats per minute. Denial about being underweight and irritability was evident on mental state examination; but she, herself, denied having disturbed body image and preoccupations with weight and appearance. She had poor insight to her illness. Other components within mental state examination were unremarkable. She was admitted with the provisional diagnosis of anorexia nervosa and treatment was initiated accordingly.

Routine laboratory investigations including hematological and biochemical parameters were within normal limits. Ultrasound of abdomen and pelvis showed normal sized adrenals and ovaries with no pathology. Further investigations were conducted to find out her endocrinological functioning resulting from long term starvation. Thyroid function tests (free T3, free T4, and TSH), serum follicle stimulating hormone (FSH) and leutinizing hormone (LH) were all within normal range. Serum cortisol was low; $48 \mathrm{ng} / \mathrm{ml}$ (reference range; 68- 223 $\mathrm{ng} / \mathrm{ml}$ ) and serum prolactin was high; $67 \mathrm{ng} / \mathrm{ml}$ (reference range; $2-29 \mathrm{ng} / \mathrm{ml}$ ). Subsequently, neuroimaging was planned. Magnetic Resonance Imaging (MRI) brain showed significantly enhancing lobulated soft tissue lesion in sellaturcica with normal sized sella and encasement of right internal carotid artery suggestive of pituitary adenoma. Final diagnosis of pituitary adenoma was then made, and neurosurgical consult was done. Neurosurgical team planned for excision of the tumor but her family refused to give consent for surgery following which she was discharged on request by the neurosurgical team. Few days later, we came to know that the patient had died at home.

\section{DISCUSSION}

Structural causes of obesity, anorexia, central hypothermia and hyperthermia, insomnia, and hypersomnia are only very rarely demonstrated. Imaging in these patients where other specific neurologic or endocrine abnormality is absent is almost always unrewarding. ${ }^{6}$ Only in rare cases an organic cause of anorexia has been recorded. ${ }^{7}$ The association between hypothalamic lesions and eating disorders is a well-known fact but the association between pituitary tumors and eating disorders is not that common. Although hypothalamic lesions are the most commonly reported neural causes of anorexia-like syndrome, most of them lack the typical psychopathology. ${ }^{8}$ Pituitary adenomas are the 
most common lesions of the pituitary gland. These may secrete prolactin, thyroid stimulating hormone (TSH), growth hormone $(\mathrm{GH})$, adrenocorticotropic hormone (ACTH), or gonadotropins. Prolactinomas most commonly present as microadenomas in premenopausal females with amenorrhea and galactorrhea. Prolactin elevation, by itself, is nonspecific and may be due to a variety of medical, neurologic, or pharmacological causes as well as pituitary adenoma, depending on serum hormone level. In males, prolactinomas may be entirely asymptomatic until visual symptoms occur, due to compression of the optic chiasma, or they may result in hypogonadotropic hypogonadism with loss of libido and impotence. Pituitary microadenomas and macroadenomas are frequently associated with a normal sella size as was evident in our case. In rare cases, brain tumors have been associated with anorexia, which frequently occurred before the etiological diagnosis was made. ${ }^{9}$

Conversely, it is not uncommon for the diagnosis of early-onset anorexia nervosa to be missed because of the relentless pursuit of an intracranial explanation for the weight loss and change in behavior. There have been a number of case reports of patients with cerebral tumors associated with symptoms suggestive of anorexia nervosa, but very few cases have completely fulfilled diagnostic criteria, and symptoms improved following treatment of the tumor. The majority of tumors were hypothalamic in origin, which accounted for the disordered eating. ${ }^{0}$ Our patient had atypical psychopathology and severe nausea and vomiting with strong family history of malignancies which should have prompted us for an early neuroimaging but was unfortunately neglected. Besides, she had low serum cortisol level which was difficult to explain. Current literature has shown high serum levels of cortisol in cases of anorexia nervosa and prolonged fasting, ${ }^{4,11}$ so could this be comorbid diagnosis? We need more researches in this field.

\section{REFERENCES:}

1. SeidenstickerJF, Tzagournis M. Anorexia nervosaClinical features and long term follow up. J Chron Dis 1968;21:361-367.

2. Sawicka N, Gryczyńska M, Sowiński J, TamborskaZedlewska M, Ruchata M. Two diagnoses become one? Rare case reportof anorexia nervosa and Cushing's syndrome. Neuropsychiatric Disease and Treatment 2013;9:431-435.

3. Saddock BJ, Saddock VA, Ruiz P. Kaplan and Saddock's Comprehensive Textbook of Psychiatry. USA: Lippincott Williams and Wilkins; 2009.

4. Gelder MG, Andreasen NC, Lopez-IborJJ,Jr, Geddes JR. New Oxford Textbook of Psychiatry. USA: Oxford University Press; 2009.

5. Winston AP, Barnard D, D'Souza G, Shad A, Sherlala $K$, Sidhu J, et al.PinealGerminoma Presenting as Anorexia Nervosa:Case Report and Review of the Literature. Int $J$ Eat Disord 2006;39:606-608.

6. Seidenwurm DJ. Neuroendocrine imaging. Am J Neuroradiol2008;29:613-615.

7. Gheorghiu M, Lisievici M, Morosan M, Ciurea AV, Coculescu M. Anorexia associated with a pineal gangliocytoma. ActaEndocrinologica (Buc) 2006;II(3):355364.

8.Uher $R$, Treasure J.Brain lesions and eating disorders. J NeurolNeurosurg Psychiatry 2005;76:852-857.

9. Kars $M$, Roelfsema $F$, Romijn JA, Pereira AM. Malignant prolactinoma: case report and review of the literature. European Journal of Endocrinology 2006;155:523-534.

10. Kumar T, Kathpal A, Longshore CT. Right Temporal Lobe Meningioma presenting as postpartum depression: A case report. Apollo Medicine 2013, http://dx.doi.org/10.1016/j.apme.2013.01.018.

11. Korbonits M, Blaine D, Elia M, Powell-Tuck J. Metabolic and hormonal changes during the refeeding period of prolonged fasting. European Journal of Endocrinology 2007;157:157-166. 